\author{
Barbara Komenda-Earle \\ ORCID: 0000-0002-5112-9336 \\ Universität Szczecin, Szczecin
}

DOI: $10.19195 / 0435-5865.143 .14$

\title{
Dephraseologische Derivate oder sog. Einwortphraseologismen? Historisch-etymologische Analysen von verbalen Einheiten
}

\begin{abstract}
s
Der Beitrag thematisiert die Problematik der dephraseologischen Derivation und die Frage der sog. Einwortphraseologismen. Unter Anwendung von etymologischen Kriterien werden diachrone Analysen von verbalen Einheiten des Typs sich (k)einen abbrechen, es jdm. angetan haben, jdm. etwas aufbinden, jdm. einheizen durchgeführt. Aus sprachhistorischer Perspektive wird der gegenwärtige Status solcher Einheiten festgelegt. Soweit die verbalen Ausdrücke Ergebnisse von regulären Metaphorisierungsprozessen darstellen und syntaktisch keine elliptischen Bildungen sind, werden sie als monolexeme Einheiten angesehen. Die metaphorischen und zugleich elliptischen Einwortausdrücke können mit dem Terminus Einwortphraseologismen im engeren (etymologischen) Sinne erfasst werden.
\end{abstract}

Schlüsselwörer: Lexik, Phraseologie, Etymologie, dephraseologische Derivation, Sprachwandel

\section{Dephraseological derivatives or so-called one-word phrases? Historical and etymological analyses of verbal units}

The paper deals with the issue of dephraseological derivation and the question of the so-called one-word phrases (German term Einwortphraseologismen). Using etymological criteria, diachronic analyses of German verbal units of the type sich (k)einen abbrechen, es jdm. angetan haben, jdm. etwas aufbinden, jdm. einheizen were carried out. The current status of such units was determined in the historical context of the language. To the extent that the verbal expressions represent the results of regular metaphorical processes and are not syntactically elliptical formations, they were regarded as mono-lexical units. The metaphoric and at the same time elliptical one-word expressions can be described with the term one-word- phrases on etymologic grounds (German term Einwortphraseologismen im engeren (etymologischen) Sinne).

Keywords: lexis, phraseology, etymology, dephraseological derivation, language history 
Barbara Komenda-Earle, Uniwersytet Szczeciński, Instytut Filologii Germańskiej, Al. Piastów 40b, 71-065 Szczecin, Polen, E-Mail: barbara.komenda-earle@usz.edu.pl

Received: 11.09.2017, accepted: 18.05.2018

\section{Einleitung}

Dephraseologische Derivation bedeutet die Entstehung von Wortbildungskonstruktionen auf der Basis eines Phraseologismus (vgl. Fleischer ${ }^{2}$ 1997: 185f.). ${ }^{1}$

Gegenstand der vorliegenden Untersuchung sind verbale Einheiten des Typs $j d m$. etwas anhängen, jdm. etwas aufbinden, aufschneiden, sich (k)einen abbrechen, es jdm. eintränken, jdm. einseifen, die sich gegenwärtig formal an der Grenze zwischen Lexik und Phraseologie platzieren.

In einer diachron angelegten Analyse wird ihre Entstehung auf der Basis von Phraseologismen, ggf. die weiterhin bestehende semantisch-formale Zusammengehörigkeit mit mehrgliedrigen phraseologischen Konstruktionen nachgewiesen und die Frage erörtert, ob sie genetisch als phraseologische Ellipsen einzustufen sind.

Ellipsen sind eines der Verfahren des semantischen Wandels, die auf der Verkürzung von Ausdrücken basieren. Historisch-semantisch sind elliptische Neubildungen dadurch zu erklären, „dass das verbleibende Wort die ursprüngliche Gesamtbedeutung auf sich konzentriert und dadurch Bedeutungswandel vollzieht“" (Nübling et al. 2010: 125).

Des Weiteren wird in Betracht gezogen, ob historisch-diachron ermittelbare phraseologische Ellipsen Einwortphraseologismen im engeren Sinne darstellen.

Bei dieser Zielvorstellung gehört sich eine erklärende Bemerkung. In historisch-diachronen Ermittlungen werden wohlüberlegt zweierlei Perspektiven der sprachhistorischen Forschungen vereinigt. Während (sprach)historische Untersuchungen jede beliebige Untersuchungen der Vergangenheitsstadien der Sprache inkludieren können und auch synchrone Betrachtungen einschließen können, werden durch die Attribuierung diachron und verstärkend historisch-diachron die Ermittlungen der Entwicklungsprozesse und -stadien der Sprache fokussiert, wobei insbesondere die Dynamik der sprachhistorischen Prozesse hervorgehoben wird.

\section{Problemstellung}

Qualitätsbestimmende Merkmale der phraseologischen Einheiten sind bekanntlich Mehrgliedrigkeit, Idiomatizität, Festigkeit/Stabilität, Lexikalisierung und Reproduzierbarkeit.

${ }^{1}$ Dephraseologische Derivation bildet eine andere Erscheinung als phraseologische Derivation (vgl. Fleischer ${ }^{2}$ 1997: 189 f.). 
Argumente für die Einführung des Terminus Einwortphraseologismus in Bezug auf deutsche Komposita bilden der idiomatische (bzw. teilidiomatische) Charakter, also Verwandtschaft in semantischer Hinsicht mit dem Kernbereich der Phraseologie sowie die Häufigkeit solcher Einheiten im Deutschen (vgl. Duhme 1995: 83; Szczęk 2004: 75; Ganzer 2008: 26).

Das wesentlichste Gegenargument stellt das formale Kriterium der fehlenden Mehrgliedrigkeit/Polylexikalität (vgl. Pilz 1981: 17; Fleischer ${ }^{2}$ 1997: 249; Ganzer 2008: 24) dar.

Der Terminus des Einwortphraseologismus ist bisher auf substantivische Komposita bezogen worden, die idiomatisch geprägt sind, d.h. in denen mindestens ein kompositioneller Bestandteil einen erkennbaren idiomatischen Charakter aufweist (vgl. Duhme 1995: 91). Beispielklassiker sind an dieser Stelle Komposita wie Blauhelm, Drahtzieher, Geldhahn, Nervenbündel, Prügelknabe.

Unter Berücksichtigung des eingangs genannten Kriteriums der dephraseologischen Derivation werden synchron zwei weitere Gruppen von substantivischen Einwortphraseologismen unterschieden:

- Einheiten mit phraseologischer Wortbildungsetymologie, die auf die ihnen zugrunde liegenden mehrgliedrigen Phraseologismen zurückführbar sind, parallele Bildungen zu Phraseologismen darstellen oder ein Ergebnis dephraseologischer Derivation sind, z.B. der Schwarzmarkt - der schwarze Markt, schwarz brennen - der Schwarzbrenner, die schwarze Fahrt-die Schwarzfahrt,

- Einheiten, die ad hoc gebildet werden, bei denen keine phraseologische Etymologie erkennbar ist, z.B. Knochenarbeit ,Ahnenforschung', Genbastelei 'Klonen' (vgl. Szczęk 2004: 79 f.).

Einen kulturell-etymologisch gestützten Vorschlag der Abgrenzung von Phraseologismen (Redensarten) ${ }^{2}$ und substantivischen Komposita unterbreitet Aichinger (2002: 27), indem er synchron durchsichtige Komposita wie Angsthase, Bücherwurm, Faulpelz, Frechdachs, Stubenhocker, die „einmalige, klar umrissene Charakterisierungen“"verkörpern, Einheiten wie Zankapfel, Pyrrhussieg, Danaergeschenk als Einwortphraseologismen gegenüberstellt, weil sich die letzteren erst aus breiteren kulturellen Kontexten erklären lassen.

Ziel des vorliegenden Beitrags ist es, das Kriterium der dephraseologischen Derivation etymologisch-diachron auszutragen und auf verbale Einheiten anzuwenden, die soweit in der Forschung als sog. Einwortphraseologismen nicht in Betracht gezogen worden sind.

2 Termini Phraseologismus und Redensart werden im vorliegenden Beitrag synonym verwendet. 


\section{Etymologische Überprüfbarkeit}

In knappster Formulierung „kann [der Etymologe] aus dem Wortschatz herausgelöste Komplexe untersuchen, deren Zusammengehörigkeit und Entwicklung auf Grundmotive und rekonstruierte Grundformen zurückführen" (Pfeifer ${ }^{6} 2003$ : VI).

Die Aufgabe von etymologischen Untersuchungen besteht darin zu prüfen, woher die lexikalischen Einheiten einer Sprache (Lexeme, Phraseologismen) kommen.

Die Frage hat jeweils zwei Seiten:

- die Bildungsgrundlage, also die Entstehung im engeren Sinne,

- die Eigenentwicklung, abgesehen von der systematischen Verwendung und dem Beginn der Geschichte des betreffenden Lexems.

Die Prinzipien werden in der Etymologie erstrangig auf Einzellexeme bezogen, sie lassen sich dennoch problemlos auch auf phraseologische Wortverbindungen übertragen. Syntaktische Fügungen wie grüner Salat, etwas auf die lange Bank schieben, also Phraseologismen werden auch von Etymologen einstimmig als Lexikalisierungen angesehen (vgl. Seebold 1984: 823, Kluge ${ }^{23}$ 1995: XIX).

Als Prinzipien der etymologischen Überprüfbarkeit wird im Folgenden Zusammengehörigkeit des Lexems/ Einwortphraseologismus zu einer früheren phraseologischen Form gesehen im Sinne:

a. der semantischen Zusammengehörigkeit, d.h. der zusammenhängenden Bedeutungsentwicklung (also gemeinsame Motivationsbasis, gemeinsames Etymon/ Benennungsmotiv),

b. der morphosyntaktischen Zusammengehörigkeit, d.h. Entstehung des gegenwärtigen Lexems/ Einwortphraeologismus infolge der elliptischen Verkürzung einer größeren syntaktischen Abfolge (der sog. derivationellen Basis) in Form von freien Wortverbindungen, anderen Phraseologismen, Sprichwörtern, phraseologischem Material etc.),

c. als drittes zusätzliches Kriterium wird die gemeinsame („,merkwürdige“ im Sinne Burgers ${ }^{4}$ 2010) Bildlichkeit mit einbezogen, das in der Auffassung gegenwärtiger Phraseologie einen der wichtigsten Beweggründe zur Untersuchung der Historizität der Phraseologismen darstellt (vgl. Burger ${ }^{4}$ 2010: 132).

Eine monolexikale Einheit wird im Folgenden auf den ihr zugrunde liegenden mehrgliedrigen Phraseologismus bzw. eine Wortverbindung mit einem gewissen Grad an Festigkeit zurückgeführt, wobei sich diachron Unterschiede in der Semantik der zusammengestellten Einheiten ergeben können. ${ }^{3}$ Der Terminus phraseologisch wird dementsprechend auf Wortverbindungen mit einem gewissen Grad an Festigkeit bezogen, der Terminus idiomatisch bezieht sich in erster Li-

${ }^{3}$ Beim Abgleich von substantivischen Einwortphraseologismen und entsprechenden mehrgliedrigen phraseologischen Einheiten auf der synchronen Ebene werden keine semantischen Unterschiede zugelassen (vgl. Szczęk 2004: 79, Anmerkung 2). 
nie auf die semantische Qualität von Wortverbindungen und Einwortausdrücken. ${ }^{4}$ Einheiten wie etwas auf die lange Bank schieben, jdm. einen Bären aufbinden sind also phraseologisch und (voll)idiomatisch, Einheiten wie der schwarze Markt, die schwarze Fahrt phraseologisch und teilidiomatisch, Einheiten wie grüner Salat phraseologisch.

\section{Verbale Einwortphraseologismen in diachroner Sicht}

Die dephraseologische Derivation ist einleuchtend bei substantivischen Komposita, die aufgrund von phraseologischen Wortverbindungen entstanden sind.

Die sog. Einwortphraseologismen bilden in dem Sinne substantivische $\mathrm{Zu}-$ sammensetzungen wie z.B. Süßholzraspler. Das Substantiv Süßholzraspler wird in übertragenem Sinne seit dem 16. Jh. verwendet, wobei es anhand der vorher bestehenden Verbalphrase Süßholz raspeln 'einer Frau in auffallender Weise schmeicheln, ihr den Hof machen, mit ihr schöntun' gebildet wird (vgl. DWB Bd. 20, Sp. 1336, Pfeifer et al. ${ }^{2} 1993 / 2017$, Stichwörter süß, Süßholz). Analoge Bildungsprozesse sind bei Brotkorbanhänger, Federstreicher, Kerbreder (vgl. Komenda-Earle 2015: 362), Bogenspucker, Dünnbrettbohrer, Haarspalter, Halsabschneider, Phrasendrescher, Possenreißer, Schwarzseher (vgl. Fleischer ${ }^{2} 1997$ : 186) nachvollziehbar.

Eismann (2010: 712) benutzt den Terminus phraseologische Wörter (nicht aber Einwortphraseologismen) in Bezug auf:

- monolexikale Einheiten mit unikalen Morphemkombinationen wie z.B. Himbeere,

- monolexikale Einheiten mit spezifischen Morphemkombinationen wie z.B. Busenfreund,

- monolexikale Einheiten, die - wie gerade die oben ermittelten Substantive des Typs Süßholzraspler - von Phraseologismen deriviert sind, z.B. Reißausnehmer, Schnippchenschlager, Bärenaufbinder.

Schwieriger als bei substantivischen Einheiten ist die Frage der dephraseologischen Derivation bei verbalen Wortschatzeinheiten zu durchleuchten, so z.B. bei Einheiten wie jdm. etwas anhängen, jdm. etwas aufbinden, es jdm. eintränken, jdm. einseifen.

Als Einwortphraseologismen könnten synchron Verben angesehen werden, die:

${ }^{4}$ Vgl. dazu auch die Stellung von Pilz (1981: 53), der vorschlägt, den Terminus Idiom ,ausschließlich als semantischen Terminus für sprachliche Einheiten jeder Größenordnung [also auch Einzelwörter] in der langue und in der parole, also auch ad hoc gebildete Neuschöpfungen, zu verwenden, in etwa gleichbedeutend mit „bildlich/metaphorisch/übertragen“. Die daran anschließende Bemerkung lautet: „Dabei stellt sich jedoch die Frage, ob neben diesen ,idiomatisch“ überhaupt noch erforderlich ist". 
- mit ihren regelmäßigen Dativ- und Akkusativergänzungen vorkommen, z.B. jdm. etwas anhängen, jdm. einseifen) sowie

- Verben, die außerdem in größeren phraseologisch gebundenen Formen auftreten, z.B. abbrechen: sich (k)einen abbrechen, aufschneiden: (gern) aufschneiden (wollen), ausstechen: jdn. ausstechen und jdn. bei jdm. ausstechen und dabei die einzige autosemantische Komponente bilden.

Das Beispiel jdm. etwas aufbinden, das synchron parallel zu dem semantisch äquivalenten Phraseologismus jdm. einen Bären aufbinden vorliegt, weist darauf hin, dass Verben dieser Art elliptische Abkürzungen phraseologischer Vollformen sein können und mit dem Terminus Einwortphraseologismen im engeren Sinne erfasst werden könnten.

In Lutz Röhrichs Lexikon der sprichwörtlichen Redensarten ${ }^{5}$ (2004) werden im Bereich der Buchstaben A-E als Stichwörter Verben wie: abbrechen phraseologisch gebunden als: sich (k)einen abbrechen, anhängen phraseologisch gebunden als: jdm. etwas anhängen, anzettelln: etwas anzetteln, aufbinden: jdm. etwas aufbinden, ausstechen: jdn. ausstechen, baldowern: etwas baldowern, becircen, einheizen phraseologisch gebunden als: jdm. (tüchtig) einheizen, eintränken phraseologisch gebunden als: jdm. etwas eintränken, einseifen phraseologisch gebunden als: jdm. einseifen aufgelistet. ${ }^{6}$

Im Folgenden gilt es, ihre dephraseologische Provenienz etymologisch nachzuprüfen.

$\mathrm{Zu}$ diesem Zweck wird ein Untersuchungsraster entwickelt, das außer gegenwärtigen Bedeutung(en) und Form(en) des Phraseologismus seine früheren

${ }^{5}$ Der Begriff der Redensarten deckt sich weitgehend mit dem Begriff der idiomatischen Redewendung: „Idiomatische Redewendungen sind solche Wendungen, deren Sinn ein anderer ist als die Summe der Einzelbedeutungen der Wörter“ (Friedrich 1976: 7, vgl. Röhrich 2004: 133). Bei den sprichwörtlichen Redensarten kommt zusätzlich der Umstand hinzu, dass es phraseologische Einheiten sind, die „die einer kulturhistorischen Herleitung bedürfen“ (Röhrich 2004: 9).

${ }^{6}$ Dass Röhrich Einzellexeme unter Phraseologismen auflistet, hat offensichtlich andere Gründe als die Reize ihrer Bildlichkeit. Davon zeugt das Zitat aus der Einleitung zum Lexikon: „Man könnte natürlich jede einfache Benennung, z.B. auch jedes originelle Schimpfwort, in eine Redensart umprägen. Es müßten dann folgerichtig auch Begriffe wie z.B. Backfisch - Twen-Teenager oder Starlet aufgenommen werden. Aber hier zeichnen sich deutlich die Grenzen und Aufgaben ab zwischen einem Wörterbuch der Umgangssprache der Gegenwart, das es ja bereits gibt und dem wir viel verdanken, und einem Wörterbuch der sprichwörtlichen Redensarten. Das bloße Einzelwort haben wir nicht aufgenommen, auch wenn es noch so bildkräftig erscheint“ (Röhrich 2004: 70). Zu dem Bereich zählen nach Röhrich (2004: 31-32) nicht Komposita wie z.B. Elefantenhochzeit, Kanonenbootdiplomatie, Puddingabitur, Hans-Guck-in-die-Luft, Stehaufmännchen, Hasenfuß, Schlitzohr, Sommerloch, Beziehungskiste, Pantoffelkino. Im untersuchten Bereich der Buchstaben A-E werden unter verbalen Einzellexemen dennoch Einheiten wie etwas checken, $j d n$ becircen, einen bimsen, $j d n$. erwischen einbezogen. Eine Analyse der Auswahlkriterien sowie Kriterien der Abgrenzung zwischen „einfachen Benennungen“ und sprichwörtlichen Redensarten im Sinne Röhrichs kann an dieser Stelle nicht angestrebt werden. 
(Voll)formen und Bedeutung(en) sowie seine Herkunft (als Ansatzpunkt der Bildlichkeit) umfasst und dadurch eine Grundlage für die Feststellung der etymologischen (semantischen und morphosyntaktischen) Verwandtschaft bietet.

Gegenwärtige Bedeutungen von den untersuchten Ausdrücken werden anhand der Quellen Röhrich (2004), DuRe $\left({ }^{2} 2002,{ }^{4} 2013\right)$ sowie www.redensartenindex.de und www.universal_lexikon.deacademic.com zusammengestellt?

Gebrauchskontexte werden anhand der Quellen dwds.de, www.redensartenindex.de, www. universal_lexikon.deacademic.com angegeben oder selbst konstruiert.

Quellen früherer Formen und Bedeutungen werden jeweils angegeben.

Falls vorhanden, werden bei der Untersuchungseinheit sinn- und formverwandte Redensarten zusammengetragen.

\section{Analyse ausgewählter Beispiele}

Die historische Entwicklung der ausgewählten Verben wird im oben beschriebenen Untersuchungsraster dargestellt:

abbrechen: sich einen abbrechen (ugs.)

(auch in der negierten Form: sich keinen abbrechen (ugs.))

(gegenwärtige) Bedeutung: 1. sich bei etw. sehr ungeschickt anstellen. 2. sich übermäßig anstrengen. 3. sich übertrieben vornehm tun

Gebrauchskontexte: Ich hoffe, du brichst dir keinen ab! Brich dir nur keinen ab! (www.redensartenindex.de, 29.08.2017) Das Mittagessen ist fertig und wir setzen uns zum Tisch!- Na, ja, dein Söhnchen bricht sich noch einen ab, als ob er eine extra Einladung bräuchte! (www. universal_lexikon.deacademic.com, 29.08.2017)

Bildlichkeit (Herkunftsbereich): Als Objekt des Abbrechens kommen zwei Möglichkeiten in Betracht. Das eine sei der Zacken an der Krone, wobei die Krone als Symbol der (eingebildeten) Vornehmheit gilt und das andere die Verzierungen an Möbelstücken. Illustrative Erläuterungskontexte lauten demgemäß: Brich dir keinen Zacken aus der Krone! (Zacken), Brich dir keine Verzierung ab! Die letzte Erklärung soll der städtischen Umgangssprache entstammen, wobei auch zuweilen um Möbel mit empfindlichen Zierteilen hätte handeln können. (Röhrich 2004: 154)

Sinn- und formverwandte Redensarten: sich eine Verzierung abbrechen (in der Bedeutung 3.), sich einen Zacken aus der Krone brechen (in der Bedeutung 2.)

anhängen: $j d m$. etwas anhängen

(gegenwärtige) Bedeutung: jdm. etw. [Übles] zuschreiben, aufbürden

${ }^{7}$ Bei der Übernahme der lexikographischen Bedeutungserklärungen von Phraseologismen aus den genannten Quellen kann die komplexe Problematik des semantischen Definierens, der Bedeutungsbeschreibung und -erklärung nicht ausführlich diskutiert werden. Zu unterschiedlichen Verfahren der Bedeutungserklärung von Phraseologismen in der lexikographischen Praxis, darunter in einsprachigen Wörterbüchern vgl. neulich Szczęk (2017), allgemeiner zu Techniken der Bedeutungsbeschreibung und -erklärung in Wörterbüchern vgl. Komenda (2004: 229 ff.). 
Gebrauchskontext: Ich soll das Geld geklaut haben? Das könnt ihr mir nicht anhängen! (www.redensartenindex.de, 29.08.2017). Man hängt dem Beamten einen Betrug an. (selbstkonstruiert)

Bildlichkeit (Herkunftsbereich): Das Bild des Ausdrucks stammt aus dem Rechtsbrauch des Mittelalters, bestimmte Missetäter wie Gotteslästerer, Schmäher, zänkische Weiber als ehrlose Menschen äußerlich zu kennzeichnen. Man hängte ihnen einen Gegenstand an, für schwerere Verbrechen einen Stein, für leichtere z.B. einen Flecken um und ließ sie ihn durch die Straßen der Stadt oder um das Rathaus schleppen. (vgl. Röhrich 2004: 291)

Ältere Vollform(en) und Bedeutungen: $j d m$. einen Schadflecken anhängen (DWB Bd. 8, Sp. 2145), jdm. einen Flecken, ein Blech, eine Krankheit, einen Schimpf, einen Spott, eine Lüge, eines, etwas anhängen. (Schowe 1994: 138f., sehr zahlreiche weitere historische Belege aus den Rechtsquellen, Wörterbüchern und der Dichtung, dabei unterschiedliche lexikalische Varianten und Vorläuferformen der Wendung bringt Schowe 1994: $131 \mathrm{ff}$. an)

Sinn- und formverwandte Redensarten: jdm. etwas ans Zeug flicken

aufbinden: $j d m$. etwas aufbinden

(gegenwärtige) Bedeutung: jdm. etwas Unwahres erzählen, weismachen, vorschwindeln

Gebrauchskontexte: Wer hat dir diese Lüge/Fabel/das Märchen/aufgebunden? Wer hat dir das aufgebunden? Man kann ihr alles aufbinden. Sie lässt sich nichts aufbinden. (dwds.de, Stichwort aufbinden, 23.08.2017)

Bildlichkeit (Herkunftsbereich): Die Redensart leitet sich von dem einfachen Bild: jemandem wird etwas auf den Ärmel aufgebunden, was dann zu einer Belästigung wird. (Röhrich 2004: 389)

Ältere Vollform(en) und Bedeutungen: jdm. etwas auf den Ärmel binden, jdm. etwas auf den Ärmel heften 'jds. Leichtgläubigkeit missbrauchen' (BWS ${ }^{7}$ 1954: 40, Röhrich 2004: 389)

Sinn- und formverwandte Redensarten: $j d m$. einen Bären aufbinden, jdm. etwas auf die Nase binden, jdm. etwas anhängen

aufschneiden: aufschneiden mit etwas, (gern) aufschneiden (wollen) (ugs. abwertend)

(gegenwärtige) Bedeutung: ,großsprecherisch übertreiben“

Gebrauchskontexte: mit seinen Erfolgen/ Heldentaten aufschneiden (dwds.de, Stichwort aufschneiden, 23.08.2017), Wenn er von seinen Erlebnissen berichtet, schneidet er immer fürchterlich auf. (www.universal_lexikon.deacademic.com, 23.08.2017)

Bildlichkeit (Herkunftsbereich): Röhrich (2004: 398) erklärt die Redensart mit dem literarischen Bild: „bei Tische so viel Reiselügen zu erzählen, daß das Brotmesser Scharten und Zacken bekommt und der Gastgeber damit das Brot sägen kann." Das Aufschneidemesser galt als Symbol des Streites und wurde in Bierstuben mit einer Glocke an der Decke befestigt, an der man läuten konnte, wenn einer eine handgreifliche Lüge erzählte. (Röhrich 2004: 399, Nübling et al. ${ }^{3}$ 2010: $125-126$ )

Ältere Vollform(en) und Bedeutungen: mit dem großen Messer aufschneiden, übertriebene Geschichten erzählen` (vgl. BWS ${ }^{7}$ 1954: 41, Röhrich 2004: 398)

Sinn- und formverwandte Redensarten: starke Stücke auftischen 
ausstechen: $j d n$. ausstechen, $j d n$. bei jdm. ausstechen

(gegenwärtige) Bedeutung: 1.jdn. verdrängen 2.jdn. übertreffen

Gebrauchskontext: Jemand hat ihn im Beruf/ bei seiner Freundin ausgestochen. Sie kleidete sich besonders gut, um alle anderen auszustechen. Sie musste ihn wieder einmal ausstechen und ein tolles Geschenk vorbereiten. (dwds.de, Stichwort ausstechen und Verwendungsbeispiele, 23.08.2017)

Bildlichkeit (Herkunftsbereich): Die Redensart stammt aus dem Turnierwesen, ist aus der älteren Vollform aus dem Sattel stechen verkürzt und dann auch auf andere Bereiche übertragen worden. Außerdem wurde sie auf den Zweikampf der Ritter zu Ehren einer Dame bezogen, wo der Unterlegene vom Sieger „ausgestochen“ wurde und als Nebenbuhler nicht mehr in Betracht kam. (vgl. Röhrich 2004: 123 f.)

Ältere Vollform(en) und Bedeutungen: $j d n$. aus dem Sattel stechen, dann auch verkürzt zu: ausstechen 'durch Stechen herausnehmen oder entfernen' (17. Jh.) (Pfeifer et al. ${ }^{2} 1993 / 2017$, Stichwort ausstechen)

Die Analyse ergibt, dass Verben jdm. etwas aufbinden, jdn. ausstechen, sich einen abbrechen, mit etwas aufschneiden, jdm. etwas anhängen auf längere, vor allem aus den älteren Sprachstufen stammende bereits phraseologisierte Wortfolgen zurückzuführen sind.

Dabei lassen sich zusammenfassend folgende Entwicklungen bei einzelnen Untersuchungseinheiten verzeichnen:

sich eine Verzierung abbrechen, sich einen Zacken aus der Krone brechen $\rightarrow$ sich einen abbrechen

semantische Entwicklung: von der wörtlichen Bedeutung 'ein Schmuckelement abbrechen' zu 'ungeschickt sein, 'sich bei etw. sehr ungeschickt anstellen', das Verb abbrechen übernimmt die Bedeutung 'ungeschickt sein', infolge späterer ironischer Übertragungen bilden sich weitere Bedeutungen der Konstruktion sich einen abbrechen u.z. 'sich übermäßig anstrengen ' und 'sich übertrieben vornehm tun' heraus,

formale Entwicklung: elliptisch verkürzt durch Wegfall der phraseologisch gebundenen Akkusativobjekte eine Verzierung, einen Zacken aus der Krone, das Reflexivpronomen sich wird beim Verb abbrechen behalten; der Gebrauch der idiomatischen Formen mit den Akkusativobjekten eine Verzierung, einen Zacken aus der Krone ist weiterhin geläufig,

einem einen Schadflecken anhängen $\rightarrow j d m$. etwas anhängen

semantische Entwicklung: von der wörtlichen Bedeutung 'jdm. etwas an den Körper, an die Kleidung anhängen' zur abstrakteren 'jdm. etwas Übles aufbürden'

formale Entwicklung: elliptisch verkürzt durch Wegfall der phraseologisch gebundenen Akkusativobjekte wie eine Krankheit, einen Schimpf, einen Spott, eine Lüge, die äußere Valenz der Wendung umfasst zuerst Bezeichnungen für Konkreta (einen Schandflecken, einen Flecken, ein Blech), dann Abstrakta (eine Krankheit, einen Schimpf, einen Spott, eine Lüge) bis die syntaktische Stelle am Verb mit dem Indefinitpronomen eines und gegenwärtig etwas aufgefüllt wird; in dieser Form wird die Wendung vollständig idiomatisiert 
jdm. etwas auf den Ärmel binden, jdm. etwas auf den Ärmel heften $\rightarrow$ jdm. etwas aufbinden

semantische Entwicklung: von der wörtlichen Bedeutung 'jdm. etwas an den Ärmel binden, heften` zu 'jdm. etwas (eine Last) aufbürden', das präfigierte Verb aufbinden übernimmt die Bedeutung 'jdm. etwas aufbürgen'

formale Entwicklung: elliptisch verkürzt, die ursprüngliche phraseologisch gebundene Lokalbestimmung auf den Ärmel fällt weg, die syntaktische Stelle wird in der idiomatisierten Wendung mit Bezeichnungen für Abstrakta wie z.B. Lügen, Geschichten aufgefüllt mit dem großen Messer aufschneiden $\rightarrow$ aufschneiden (mit etwas), (gern) aufschneiden
(wollen)

semantische Entwicklung: von der wörtlichen Bedeutung 'den Braten bei Tisch (also in gewisser Öffentlichkeit) aufschneiden' zu 'angeben, prahlen', das präfigierte Verb aufschneiden übernimmt die Bedeutung 'angeben, prahlen' und behält die ursprüngliche Valenz

formale Entwicklung: elliptisch verkürzt, die Modalbestimmung mit dem großen Messer fällt weg, die syntaktische Stelle wird in der idiomatisierten Wendung mit Bezeichnungen für Abstrakta wie z.B. Erfolgen, Geschichten, Heldentaten aufgefüllt; eine Variante des Idioms bildet (gern) aufschneiden (wollen), die eine fakultative Modalbestimmung gern und die das fakultative Modalverb wollen enthält,

jdn. aus dem Sattel stechen $\rightarrow$ jdn. ausstechen

semantische Entwicklung: von der wörtlichen Bedeutung 'jdm. aus dem Sattel (im Ritterkampf) stechen' zu 'jdn. verdrängen', und dann noch einmal zu der noch abstrakteren Bedeutung 'jdn. übertreffen'; das präfigierte Verb ausstechen übernimmt die Bedeutung der ursprünglichen konkret referierenden Phrase 'jdm. aus dem Sattel stechen'

formale Entwicklung: elliptisch verkürzt durch Wegfall der ursprünglichen Komponente aus dem Sattel mit konkreter Bedeutung, das Verb stechen wird mit aus- präfigiert, womit sich auch die äußere Valenz der idiomatisierten Wendung ändert; die syntaktisch erweiterte Variante der

Wendung lautet jdn. bei jdm. ausstechen.

Bei den Verben $j d m$. etwas aufbinden, jdn. ausstechen, sich einen abbrechen, mit etwas aufschneiden, jdm. etwas anhängen wird jeweils:

a. ein semantischer Zusammenhang zwischen der Bedeutung der heutigen verbalen Einheit und ihren Vorläuferformen nachgewiesen, d.h. die zusammenhängende Bedeutungsentwicklung (also gemeinsame Motivationsbasis, gemeinsames Etymon/ Benennungsmotiv),

b. eine elliptische Verkürzung der historisch vorausgehenden größeren morphosyntaktischen Abfolgen festgestellt, die zugleich die sog. derivationelle Basis für heutige Lexeme bilden.

Insoweit formale Zusammenhänge zwischen untersuchten Verben und ihren bereits phraseologisierten mehrgliedrigen Vorläuferformen sowie die semantische Gemeinsamkeit (einschließlich der Schlüssigkeit der Bedeutungsentwicklung) der vorausgehenden und gegenwärtigen Formen nachgewiesen sind, besteht eine ausreichende Grundlage für die Feststellung der etymologischen (semantischen und morphosyntaktischen) Verwandtschaft und der dephraseologischen Derivation. 
Bei den Ausdrücken jdm. etwas aufbinden und mit etwas aufschneiden konnten auch phraseologische Parallelen, also eng sinn- und formverwandte Wortverbindungen (vgl. jdm. einen Bären aufbinden, jdm. etwas auf die Nase binden und starke Stücke auftischen) ermittelt werden. Das Vorhandensein von phraseologischen Parallelen ist ein zusätzlicher starker Hinweis auf die dephraseologische Derivation.

Die elliptischen und zugleich metaphorischen Einwortausdrücke können mit dem Terminus Einwortphraseologismen im engeren (etymologischen) Sinne erfasst werden. ${ }^{8}$

Solche Entwicklungen lassen sich dagegen bei Verben etwas anzetteln, jdn. beuteln, etwas baldowern, jdn. becircen, jdm. einheizen, jdm. einseifen nicht ermitteln.

Das Verb anzetteln - bei Röhrich als Redensart bezeichnet - war ursprünglich ein fachsprachlicher Ausdruck der Webersprache und bedeutete: 'den Anfang eines Gewebes herrichten, die Fäden aufspannen', wobei Zettel die 'Längsfäden des Gewebes ${ }^{6}$ hießen, die zum Beginn des Webens aufgezogen werden (BWS ${ }^{7} 1954$ : 34, Röhrich 2004: 326, DUW Stichwort anzetteln). Die metaphorische Bedeutung 'etwas anstiften, etwas anspinnen' bildet sich bereits im 15. Jh. heraus (vgl. Pfeifer et al. ${ }^{2}$ 1993/2017, Stichwort Zettel). Das Verb wird seit sehr frühen Belegen auf Abstrakta bezogen und wird im positiven und negativen Sinne gebraucht, vgl. z.B. meine rache sei lust und freude an dem handel, den ich nun anzetteln will. (Klinger); eine verschwörung anzetteln (zit. nach DWB Bd. 1, Sp. 526), Krieg und Hader, Heil anzetteln (Maaler 1561, zit. nach Röhrich 2004: 326).

Die Bedeutung des Verbs beuteln - bei Röhrich redensartlich als (arg) beuteln aufgenommen - änderte sich in der Vergangenheit infolge einer Metaphorisierung und Bedeutungserweiterung. Das Verb beuteln bedeutete ursprünglich 'einen Beutel (mit Mehl) schütteln', 'einen Beutel schlagen'. Die ursprünglichen Inhalte 'einen Beutel (mit Mehl) schütteln', 'einen Beutel schlagen' wurden demnächst auf Menschen bezogen. Diese Bedeutung verzeichnet auch Röhrich (2004: 720) im Lexikon der sprichwörtlichen Redensarten als ' jdn. tüchtig mitnehmen, eigentlich ihn mächtig durchschütteln’. Von der Bedeutung ‘jdn. schlagen, schütteln’ wurde im nächsten Schritt metaphorisch die Bedeutung 'jdm. Geld abnehmen' abgeleitet (vgl. Pfeifer 6 2003: 129, Pfeifer et al. ${ }^{2}$ 1993/ 2017, Stichwort Beutel).

Das Verb baldowern ist eine Ableitung von dem Substantiv Baldower, das aus dem Jiddischen (jidd. baldốwer 'der in Rede Stehende, der Betreffende') oder He-

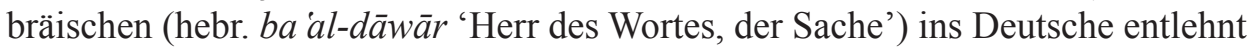
worden ist und im Mittelalter als Euphemismus für 'jmdn., dessen Namen man nicht nennen will (meistens den Teufel)' stand.

${ }^{8}$ Gleichzeitig wird zugegeben, dass bei synchroner Betrachtung der untersuchten Einheiten der Terminus nicht nötig ist. 
Das Verb gelang demnächst in den berlinerischen Jargon und anschließend in die Umgangssprache (19. Jh.) mit der Bedeutung ,auskundschaften, nachspüren' (vgl. Kluge 231995: 74, Pfeifer et al. ${ }^{2} 1993 / 2017$, Stichwort baldowern, Röhrich 2004: 495).

In dieser Bedeutung bleibt es bis heute erhalten, vgl. beispielhafte Gebrauchskontexte:

Er unterstellte: 'Die Flüchtlinge können baldowern. (Süddeutsche Zeitung, 28.04.1994, dwds

Verwendungskontexte, 24.08.2008)

Versonnen blättert Grendel in der "Paris Turf", baldowert seine Prix-d 'Amerique-Wette aus. (Bild, 28.01.2005, dwds Verwendungskontexte, 24.08.2008)

Bei den Verben arg beuteln und baldowern handelt es sich nicht um dephraseologische Derivate elliptischer Art und nicht um Einwortphraseologismen. Gegenwärtige Bedeutungen der beiden Verben stellen aus historischer Sicht Ergebnisse der metaphorischen Verschiebung der ursprünglichen, konkreten Bedeutungen von einzelnen, nicht phraseologisch gebundenen monolexikalen Einheiten und sind Ergebnisse von regelmäßigen metaphorischen Verfahren.

Das Verb becircen 'betören, verführen' ist eine Präfixableitung nach dem Namen der altgriechischen mythischen Zauberin Circe und ihren Verführungskünsten. Im Deutschen wird es seit dem 20 Jh. verzeichnet (vgl. Kluge 231995: 88, Röhrich: 2004: 610) und ist ebenfalls kein Fall der dephraseologischen Derivation.

Die Bedeutung des Verbs einseifen phraseologisch gebunden als: jdm. einseifen ist Ergebnis der metaphorischen Übertragung. Ursprünglich soll mit einseifen die Fachkunst der Friseure gemeint sein und dann die Redegewandtheit der Fachleute und Händler beim Kundengewinn (vgl. Röhrich 2004: 1457). Hier handelt es sich auch um keine dephraseologische Derivation.

Eine metaphorische Bedeutungsentwicklung hat auch beim Verb einheizen stattgefunden. Die ursprüngliche Bedeutung 'heizen, Feuer machen' (14. Jh.) wurde in die Bedeutung 'jdm. zusetzen, jdm. Vorwürfe machen' übertragen (vgl. Pfeifer et al. ${ }^{2} 1993 / 2017$, Stichwort heizen), vgl. einige gegenwärtige Verwendungsbeispiele:

Was sollen wir ihr noch einheizen! (dwds, Kernkorpus 1900-1999, 23.08.2017)

Ich werde ihm schön einheizen. (dwds, Verwendungskontexte, 23.08.2017)

Röhrich (2004: 1445) registriert das Verb in der phraseologisch gebundenen Form jdm. (tüchtig) einheizen, dwds.de (29.08.2017) gibt als Gebrauchsbeispiele von einheizen phraseologische Formen jdm. (gehörig, schwer, ein bisschen) einheizen und jdm. durch reichliche Arbeit, durch Antreiben einheizen an.

Abschließend werden sprachhistorische Entwicklungen bei den Wortverbindungen der vollidiomatischen Wortverbindungen es jdm. angetan haben 'jdn. ganz 
für sich einnehmen' und jdm. etwas eintränken ‘jdm. etwas beibringen, es jdm. vergelten' kurz beleuchtet.

Die Wortverbindung es jdm. angetan haben kommt in den frühesten Belegen in der heutigen phraseologisch gebunden idiomatischen Form vor, vgl. der ihnen solche schmach anthat (Fischart 1525), es ist mir angethan worden (Stieler 1691) (beide Belege nach Röhrich 2004: 91).

Es jdm. angetan haben bedeutet ursprünglich 'ihn bezaubert, verhext haben', wobei antun eine verhüllende Ausdrucksweise für das Schaden- oder Liebeszaubern der Hexen ist und das unbestimmte es verhüllend für 'das, was die Hexen einem antun' steht. Durch den Einsatz von zwei verhüllenden Elementen wird die Ausdrucksweise intensiviert (vgl. BWS ${ }^{7}$ 1954: 34; Röhrich 2004: 91).

Trotz ihres Einwortcharakters (eine autosemantische Komponente antun) wird die Wortverbindung von der heutigen phraseologischen Forschung als phraseologische Einheit anerkannt (vgl. der Eintrag in DuRe ${ }^{2} 2002,{ }^{4} 2013: 45$ ).

Analoge Etymologie liegt bei dem nicht mehr geläufigen Verb eintränken, phraseologisch gebunden als: jdm. etwas eintränken vor. Die ursprüngliche Bedeutung des Verbs war, jdm. einen Trank zu trinken geben', das unbestimmte es (dann etwas) stand in der Wortverbindung ebenso verhüllend für etwas Schlimmes, vor allem für das zu trinkende Gift (Röhrich 2004: 1459).

\section{Fazit}

Die präsentierte etymologisch abgesicherte Methode lässt die semantisch-formale Zusammengehörigkeit mit mehrgliedrigen phraseologischen Konstruktionen bei den Verben jdm. etwas anhängen, jdm. etwas aufbinden, aufschneiden, sich einen abbrechen nachweisen und sie als phraseologische Ellipsen einzustufen.

Im Falle von anderen Verben, wie etwas anzetteln, jdn. beuteln, baldowern, die ebenfalls als ,Träger von Konzepten [...], die in Form eines einzelnen Begriffs komplizierte Handlungsabläufe oder Situationen beschreiben“ (Duhme 1995: 85) betrachtet werden können, liegt keine dephraseologische Derivation vor, so dass die Anwendung des Terminus des Einwortphraseologismus in diesen Fällen im o.g. Sinne nicht zu begründen ist.

Als Sonderfälle werden die Wendungen es jdm. angetan haben, es jdm. eintränken ausgewiesen, die jeweils zwar nur eine autosemantische Komponente enthalten, etymologisch jedoch als phraseologische vollidiomatische Mehrworteinheiten erklärbar sind. 
Eine Erweiterung des Terminus Einwortphraseologismus auf verbale Einheiten des Typs (arg) beuteln, (aus)baldowern, becircen würde die Einbeziehung der kulturell-diachronen Kriterien im Sinne Aichingers (2002) bedeuten. Die bekannte technische Frage würde dann allerdings lauten, wie weit die Phraseologieforschung reichen will und inwieweit die phraseologischen Untersuchungsmethoden und -ziele durch Gesichtspunkte anderer Wissenschaften (in diesem Falle der Kulturforschung und Folkloristik) beeinflusst werden wollen.

\section{Literatur}

\section{Quellen}

Aichinger, Friedrich (2002): Über die Schulter geschaut. Lebendige Sprache. Alte und neue Redewendungen. Eigenverlag Leonberger.

Borchardt, Wilhel / Wustmann, Gusta / Schoppe, Georg (1888/ $\left.{ }^{7} 1954\right)$ : Die sprichwörtlichen Redensarten im deutschen Volksmund nach Sinn und Ursprung erläutert. (neu bearbeitet von Alfred Schimer). Leipzig. (= BWS $\left.1888 /{ }^{7} 1954\right)$

Deutsches Wörterbuch von Jacob und Wilhelm Grimm. 16 Bände in 32 Teilbänden (1854-1961). Leipzig: Verlag von S. Hirtel, Quellenverzeichnis Leipzig 1971. http://www.woerterbuchnetz. de/DWB/. (= DWB)

Dudenredaktion (Hrsg.) (22002, $\left.{ }^{4} 2013\right)$ : DUDEN. Redewendungen und sprichwörtliche Redensarten. Bearbeitet von Brigitte Alsleben und Werner Scholze-Stubenrecht. (= Der Duden in 12 Bänden, Band 11). Mannheim u.a.: Dudenverlag. (= DuRe $\left.{ }^{2} 2002,{ }^{4} 2013\right)$

http://www.dwds.de (Zugang 23.-29.08.2017)

Eismann, Wolfgang (2010): Phraseologische Gemeinsamkeiten der Sprachen Europas. In: Hinrichs, Uwe (Hrsg.): Handbuch der Eurolinguistik. Wiesbaden. S. 711-727.

Friederich, Wolf ( $\left.{ }^{2} 1976\right)$ : Moderne deutsche Idiomatik. Alphabetisches Wörterbuch mit Definitionen und Beispielen. Ismaning: Hueber.

Kluge, Friedrich $\left({ }^{23} 1995\right)$ : Etymologisches Wörterbuch der deutschen Sprache. 23. erweiterte Auflage (bearbeitet von Elmar Seebold). Berlin/New York.

Pfeifer, Wolfgang et al. (21993/2017): Etymologisches Wörterbuch des Deutschen. Digitalisierte und von Wolfgang Pfeifer überarbeitete Version im Digitalen Wörterbuch der deutschen Sprache http://www.dwds.de (29.08.2017).

Pfeifer, Wolfgang ( $\left.{ }^{6} 2003\right)$ : Etymologisches Wörterbuch des Deutschen. München: Deutscher Taschenbuchverlag.

Röhrich, Lutz (2004): Lexikon der sprichwörtlichen Redensarten. Digitale Bibliothek 42. Berlin. Wörterbuch der deutschen Gegenwartssprache (2013-2018, im Aufbau). www.dwds.de. (= DWDS) www.universal_lexikon.deacademic.com (Zugang 23.-29.08.2017).

\section{Sekundärliteratur}

Burger, Harald ( $\left.{ }^{4} 2010\right)$ : Phraseologie. Eine Einführung am Beispiel des Deutschen. 4. neu bearbeitete Auflage. Berlin. 
Duhme, Michael (1995): Lauschangriff und Rollkomando - ,Einwortphraseologismen “ in der Pressesprache am Beispiel des Nachrichtenmagayins FOCUS. In: Baur, Ruprecht S. / Chlosta, Christoph (Hrsg.): Von der Einwortmetapher zur Satzmetapher. Akten des westfälischen Arbeitskreises Phraseologie-Parömiologie. Bochum. S. 83-93.

Fleischer, Wolfgang ( $\left.{ }^{2} 1997\right)$ : Phraseologie der deutschen Gegenwartssprache. Tübingen.

Friederich, Wolf ( $\left.{ }^{2} 1976\right)$ : Moderne deutsche Idiomatik. Alphabetisches Wörterbuch mit Definitionen und Beispielen. Ismaning.

Ganzer, Dinara (2008): Deutsche Phraseologismen mit Personennamen. Lexikographischer Befund und textueller Gebrauch. Hamburg.

Komenda, Barbara (2004): Methoden der Bedeutungsbeschreibung. Das Problem der semantischen Definition anhand von einsprachigen deutschen und polnischen Wörterbüchern, in: Germanistisches Jahrbuch Polen. DAAD Convivium. Bonn. S. 223-244.

Komenda-Earle, Barbara (2015): Sprachhistorische Entwicklungsprozesse der Idiomatik. An Beispielen von realhistorisch motivierten verbalen Idiomen des Deutschen. Baltmannsweiler.

Nübling, Damaris (in Zusammenarbeit mit Antje Dammel, Janet Duke, Renata Szczepaniak) (32010): Historische Sprachwissenschaft des Deutschen. Eine Einführung in die Prinzipien des Sprachwandels. Tübingen.

Pilz, Klaus Dieter (1981): Phraseologie. Redensartenforschung. Stuttgart.

Schowe, Ulrike (1994): Mit Haut und Haar. Idiomatisierungsprozesse bei sprichwörtlichen Redensarten aus dem mittelalterlichen Strafrecht. Frankfurt am Main/Berlin.

Szczęk, Joanna (2004): Einwortphraseologismen und ihr Verhältnis zur Phraseologie (am Beispiel des Deutschen und des Polnischen). In: Földes, Csaba / Wirrer, Jan (Hrsg.): Phraseologismen als Gegenstand sprach- und kulturwissenschaftlicher Forschung. Akten der Europäischen Gesellschaft für Phraseologie (EUROPHRAS) und des Westfälischen Arbeitskreises „Phraseologie/Parömiologie" (Loccum 2002). Hohengehren. S. 75-85.

Szczęk, Joanna (2017): Phraseologische Semantik in der lexikographischen Praxis (am Beispiel des Sprachenpaares Deutsch und Polnisch. In: Lipczuk, Ryszard / Lisiecka-Czop, Magdalena / Ramers, Karl Heinz (Hrsg.): Sprache und Wörterbücher in Theorie und Praxis. Lexikografische und textlinguistische Fragestellungen. S. 213-226.

Seebold, Elmar (1984): Etymologie. In: Besch, Werner / Reichmann, Oskar / Sonderegger, Stefan (Hrsg.): Sprachgeschichte. Ein Handbuch zur Geschichte der deutschen Sprache und ihrer Erforschung. Erster Halbband. Berlin/New York. S. 823-833.

Germanica Wratislaviensia 143, 2018

(C) for this edition by CNS 